\title{
Le discours rapporté comme effet de montage du discours citant et du segment citationnel. Contribution à l'étude du discours journalistique ${ }^{1}$
}

\author{
Elżbieta Biardzka \\ Université de Wrocław \\ ebiardzka@,wp.pl
}

\section{Cadre théorique, objectifs et méthode de travail}

Le décalage entre le nombre toujours modeste des formes (patterns) de discours rapporté consacrées par la vulgate et la diversité des pratiques discursives du discours rapporté (désormais DR) reste toujours aussi surprenant qu'inexplicable, même si l'on en appelle à la conception élargie du rapport de la parole d'autrui ${ }^{2}$.

Dans cette étude, nous nous proposons un retour aux données empiriques qui nous permettra de repérer et de décrire un dispositif sémantico-énonciatif et grammatical qui est, d'après nous, à l'origine de la prolifération de pratiques du DR (codifiées et non codifiées ) particulièrement répandues dans la presse écrite. Ceux qui lancent les opinions que la presse en général, y compris la presse écrite, est un réservoir d'exemples « déviants » et, dans ce sens, moins intéressants scientifiquement, ne sont pas rares du tout (cf. à ce propos Rosier, 2002 : 27-28 et Hausmann, $2000: 199,207)$. Franz Josef Hausmann (2000 : 199) parle d'«un extraordinaire théâtre de liberté langagière » qui se joue dans la presse dont les emplois échappent souvent aux modèles linguistiques formalisés. Ainsi, le langage de presse est méprisé et «boudé » aussi souvent qu'injustement ${ }^{3}$. Nous ne partageons pas ce point de vue, par contre nous souscrivons entièrement à l'opinion de ceux qui soulignent que la presse écrite renferme des emplois qui sont tout d'abord «légitimes », ensuite récurrents, et surtout diffusés tous les jours à des milliers d'exemplaires (Rosier, 2002 : 27-28). Le discours journalistique, qu'on le veuille ou non, institue de nouveaux modes d'expression et participe activement à les faire passer dans l'usage courant. Ainsi, loin de se révéler inutile ou scientifiquement secondaire, fonder une recherche sur un corpus de presse nous semble entièrement justifié et prometteur. Qui plus est, la presse véhicule souvent, et peut-être même avant tout, des représentations de ce qu'on appelle le discours de l'Autre. A l'origine, le journaliste se trouve déjà en présence d'un discours. Il n'a pas d'accès direct aux événements, il ne décrit pas ce qu'il a $v u$ mais plutôt ce qu'il a entendu dire soit à propos de l'événement même, soit à propos des discours déjà tenus. Nécessairement, le journaliste affronte sans cesse des discours sur quelque chose pour produire un discours à ce propos pour les lecteurs. Les discours rapportés ne sont que l'effet de la position du rapporteur dans une situation de communication particulière. Attirés par ces aspects de l'écriture journalistique, nous n'avons pas hésité à calculer nos résultats de recherche sur l'analyse d'un corpus d'à peu près 1500 exemples tirés des journaux Le Monde, Libération et Le Figaro ${ }^{5}$.

Conformément à l'approche communément admise dans la recherche actuelle ${ }^{6}$, nous considérons le DR comme une séquence textuelle binaire, embrassant deux segments distincts: le discours citant (désormais DC) qui verbalise les données situationnelles de l'énonciation primaire et le segment citationnel (Cit) qui représente les paroles. Compris de la sorte, le DR peut se figurer sous l'équation suivante : DR $=\mathrm{DC}$ + Cit.

Nous envisageons le DC et la Cit comme des sortes de briques, mettons comme des Lego, qui entrent dans plusieurs combinaisons possibles pour donner naissance aux séquences du DR ${ }^{7}$. Pour étudier le mécanisme qui les engendre, il faut inventorier et décrire les propriétés formelles et sémanticoénonciatives des segments DC et ensuite des segments Cit. Faute de place, nous omettons ici la description des critères qui nous ont servi à identifier et délimiter les deux segments qui composent le $\mathrm{DR}^{8}$. 
L'inventaire des formes grammaticales que peut revêtir le DC embrasse dans notre corpus 6 cas de figures : la phrase introductive ${ }^{9}$ (non autonome, non complète, elle est soit indépendante, soit régissante), la phrase autonome (complète), l'incise, l'interrogation, les syntagmes en «selon A », ensuite d'autres syntagmes, comme les syntagmes nominaux, adjectivaux (avec adjectif souvent adverbial), représentant différents cas d' « incomplétudes » formelles et de réductions.

La Citation, terme générique dans notre étude, recouvre trois types d'occurrences suivant l'attitude que le rapporteur prend par rapport à l'énoncé original. Les Citations reproductions (citations «fidèles ») présentent les propos évoqués dans un acte d'énonciation rapportée comme reproduits sans aucune intervention dans la textualité/matérialité du message. Les Citations reformulations «racontent » un acte de parole autre, traitent le message plus ou moins librement, résument certains éléments jusqu'à supprimer toute indication sur la matérialité du message et signaler seulement qu'il y a eu un acte de parole. Les Citations mixtes empruntent certains traits aux Citations reproductions, certains autres traits aux Citations reformulations.

Les relations syntaxiques entre les deux segments du DR dépendent de la forme de DC et se définissent par deux cas de figure. Soit le journaliste rapporte les paroles de différentes personnes sans les assimiler à la syntaxe de son propre énoncé, soit il les intègre dans son discours conformément aux règles de la syntaxe. Dans le premier cas, nous avons affaire à une combinatoire libre, dans le second cas, il s'agit d'une combinatoire contrainte.

Dans la combinatoire libre, le DC et la Cit sont syntaxiquement (et typographiquement) séparés. La séquence du DR se caractérise par une rupture syntaxique ${ }^{10}$ : le DC est en quelque sorte «ajouté » à la Cit qui ne semble pas limitée par les formes linguistiques que peut revêtir celui-ci. La combinatoire libre va au delà des limites de la phrase. Le rapporteur journaliste est libre de choisir l'emplacement des DC : leur position par rapport aux Cit n'est pas syntaxiquement contrainte ${ }^{11}$. Pour décrire ce type de fonctionnement du DR, on peut parler de DC transphrastique.

Dans la combinatoire contrainte, le DC et la Cit sont syntaxiquement liés. La séquence du DR qui résulte de la combinatoire contrainte est homogène du point de vue sémiotique et syntaxique, mais elle est hybride du point de vue énonciatif : elle abrite des contenus provenant de deux locuteurs distincts ${ }^{12 .}$ Ainsi, c'est seulement le segment DC qui contient les propos propres au rapporteur-journaliste. La jonction des $\mathrm{DC}$ et des Cit se fait à l'intérieur de la phrase qui est grammaticalement finie et autonome. Le DC est alors intra-phrastique parce qu'il est syntaxiquement soudé à la Cit et non pas «ajouté », comme dans la combinatoire libre.

\section{Combinatoire libre}

Les séquences libres du DR relevées dans notre corpus se laissent répertorier d'abord selon quatre positions principales que peut prendre le DC par rapport à la Cit : DC antéposé (DC+Cit), DC postposé ${ }^{13}$ $(\mathrm{Cit}+\mathrm{DC}), \mathrm{DC}$ intercalé dans la Cit $(\mathrm{Cit}+\mathrm{DC}+\mathrm{Cit})$ et $\mathrm{DC}$ encadrant la Cit $(\mathrm{DC}+\mathrm{Cit}+\mathrm{DC})$.

Dans le cadre de chacun de ces schémas distributionnels, on peut essayer de montrer comment les types grammaticaux des segments DC se combinent avec les types de segments Cit pour s'ouvrir aux séquences du DR respectives. Puisqu'il n'est pas possible d'étudier ici tous les cas, prenons comme exemple d'abord le premier schéma $\mathrm{DR}=\mathrm{DC}+\mathrm{Cit}$.

Les DC antéposés prennent la forme d'une phrase introductive, en l'occurrence d'une phrase complète qui peut parfois prendre la modalité interrogative. Ces DC peuvent se figurer aussi en syntagmes du type "selon A ». Parmi les types grammaticalement possibles de DC journalistiques, seule l'incise semble exclue de la position initiale dans la combinatoire des séquences du DR ${ }^{14}$. Les DC libres ouvrants se combinent dans les articles de journaux aussi bien avec des Cit reproductions qu'avec des Cit reformulations et Cit mixtes.

Un simple calcul nous indique que la combinatoire de cinq types grammaticaux de DC avec trois types de Citations aboutit à quinze différentes pratiques discursives, dont la majorité, à quelques exceptions près, 
sont attestées dans notre corpus. Certaines d'entre elles, peu nombreuses, ont acquis le statut de formes du DR enregistrées dans les grammaires. D'autres emplois, bien attestés et intervenant régulièrement dans notre corpus sont ignorés par la vulgate, et leur statut paraît donc ambigu. La combinatoire que nous nous sommes proposée de décrire se visualise comme suit :

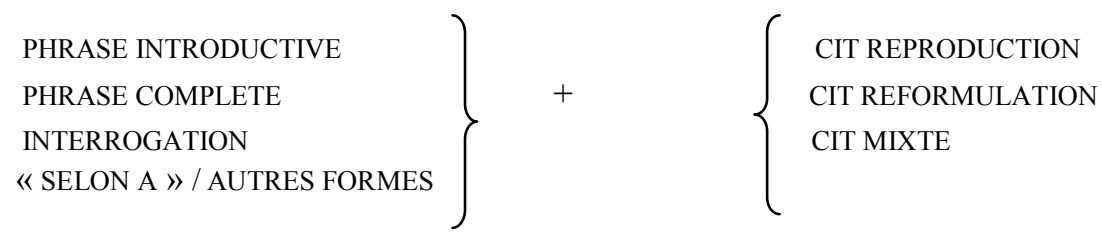

Le schéma distributionnel libre du type DC + Cit est représenté dans le corpus par le montage de la phrase introductive ou complète avec les trois types de Cit : reproduction, reformulation et Cit mixte :

PHRASE INTRODUCTIVE / COMPLETE/INTERROGATION $+\left\{\begin{array}{l}\text { CIT REPRODUCTION } \\ \text { CIT REFORMULATION } \\ \text { CIT MIXTE }\end{array}\right.$

Comme nous l'avons remarqué supra, l'ordre des segments du DR dans la combinatoire libre n'est pas figé. Ainsi, dans cette étude, nous nous choisi d'examiner également les assemblages du DR dont l'ouverture est assurée par la Cit suivie d'un DC incarné par une incise :

$\left.\begin{array}{l}\text { CIT REPRODUCTION } \\ \text { CIT REFORMULATION } \\ \text { CIT MIXTE }\end{array}\right\}+$ INCISE

\subsection{Phrase introductive/complète + Citation reproduction}

Nombre de séquences du corpus illustrent bien l'une des formes consacrées par les grammaires : celle du discours direct (DD) :

(1) Une femme, qui s'est arrêtée, s'indigne : «J'ai honte pour vous. N'importe qui a le droit de construire un lieu de prière n'importe où, même ici . " (Libération, samedi $11 /$ dimanche 12 septembre $10 / 4$ )

Parmi les modes de représentation du discours autre dans un discours, les formes du DD sont présentées dans les grammaires comme syntaxiquement simples et fidèles sur le plan sémantico-énonciatif : le DD citerait d'une manière formellement simple la matérialité textuelle du discours autre selon la formule: Il dit : «abc ». Cependant, au plan sémantique et énonciatif, le DD est hétérogène et dichotomique. Il juxtapose en effet deux énonciations: énonciation pour ainsi dire rapportante et énonciation rapportée. Les modalités d'énonciation se juxtaposent donc aussi: la séquence au DD peut renfermer par exemple à la fois une assertion et une interrogation. Les déictiques du segment DC et ceux de la Cit s'interprètent en fonction de deux situations d'énonciation distinctes et différentes: le "je » du DC renvoie au locuteur tandis que dans la Cit, la référence de ce déictique se calcule en fonction de l'énonciation rapportée : ainsi, dans une même phrase, un déictique peut avoir plusieurs valeurs. Rappelons de même la rupture sémiotique propre au DD. Dans le segment DC, le rapporteur journaliste fait usage de ses mots à lui 
pour décrire la situation d'énonciation, mais dans la seconde partie, dans la Cit reproduction, il montre, d'une façon supposée fidèle, la parole d'autrui : il fait mention des mots du message qu'il rapporte (Authier-Revuz, 1992 : 39-40). Ainsi, comme le remarque Authier-Revuz (1992 : cf. surtout 38 ; 1993), la syntaxe du DD n'est ni régulière, ni simple, ni "normale ». Elle se caractérise au contraire par une rupture marquée entre les deux segments désignés dans cette étude comme DC et Cit. Si on se propose donc de considérer le DD comme une structure syntaxique, il n'est qu'une juxtaposition de deux constructions quasiment indépendantes: la structure grammaticale de la partie citée (Cit) n'est limitée par aucune contrainte venant du côté de la partie citante (DC). De ce point de vue, le DD est une séquence prototypique du DR en combinatoire libre.

Les frontières syntaxiques qui séparent la Cit du DC sont «tranchées » et potentiellement « infranchissables » comme le dirait Bakhtine (1977 : 167-168). Les paroles d'autrui peuvent donc être protégées d'une infiltration de la part du locuteur qui les cite. Ce potentiel d'indépendance entre les segments du DD n'est guère exploité dans la pratique discursive des journaux : la Cit reproduction est souvent précédée dans la séquence par une phrase complète, et non pas par une structure « introductive » (donc incomplète) quelconque comme le prévoit le discours des grammaires. Le journaliste fournit en fait au lecteur un résumé, sinon une interprétation, des paroles évoquées, avant même d'en arriver à leur reproduction. Dans certains autres contextes, la Cit reproduction est précédée par un DC-question dont l'énonciateur est rarement explicite. En voici un exemple:

(2) Jean-François Rey, président du principal syndicat de spécialistes libéraux (UMESPE-CSMF), reconnaît que les patients vont devoir payer : " L'accord $d u 10$ janvier n'est pas satisfaisant mais dans la mesure où l'assurance maladie n'a pas les moyens financiers de rémunérer notre temps et notre valeur il faut des espaces de liberté tarifaire pour $30 \%$ à $40 \%$ des actes des médecins. Tous les Français ont un portable, ils peuvent bien payer un peu plus leurs soins. » (Le Monde, vendredi, 21 février 03/7)

Le journaliste-rapporteur, convoqué par le contrat de communication, se concentre sur différents aspects de l'énoncé rapporté : il montre les propos et s'efforce de guider leur lecture. Avant de les citer dans sa matérialité, il les reformule souvent dans le DC sous forme du discours narrativisé ou même du discours indirect (2).

Cela veut dire que le journaliste ne se limite pas à rapporter textuellement les paroles : tantôt il résume, tantôt il complète, tantôt il confirme, tantôt il désavoue les énoncés cités. A ce propos, Bakhtine expliquerait certainement que le journaliste-rapporteur manifeste une appréhension active par rapport à l'énoncé qu'il cite car «celui qui appréhende l'énonciation d'autrui n'est pas un être muet, privé de la parole » $(1977: 165)$. Au contraire, «c'est un être rempli de paroles intérieures », orienté activement envers la parole d'autrui. La parole va à la parole : le discours intérieur et le discours appréhendé de l'extérieur effectuent une jonction et donnent fruit au niveau diégétique des séquences du DR. Nous dirions plutôt pour notre part que les rapporteurs du Monde semblent s'intéresser moins aux événements qu'aux discours tenus sur ces événements.

\subsection{Phrase introductive/complète + Citation reformulation}

Cependant, nous avons repéré dans notre corpus des occurrences du DR où la phrase introductive caractéristique du DD, dotée d'un verbe de parole (ou de son équivalent) et terminée par les deux-points introduit la Cit que nous avons qualifiée de reformulation car elle n'est pas typographiée. Les verbes de parole (VP) ou leurs équivalents nominaux (nomina dicendi) ont un sens de résumés d'opinions ou de dires, ou tout au moins, signalent que les propos sont évoqués « en substance ». La phrase introductive est parfois remplacée, comme en (3), par une phrase complète simplement terminée par un point. En voici quelques exemples (les DC sont soulignés, les VP/leurs équivalents sont en italique) :

(3) Pour calmer les esprits, le premier secrétaire du parti, Francois Hollande, $a$ alors défini la ligne de conduite à tenir : l'Internationale socialiste - l'amitié - ne compte 
pas par rapport aux principes tels que la défense des droits de l'homme. (Le Monde, vendredi, 7 mars $03 / 4$ )

(4) Nigel Roberts, le directeur de la Banque mondiale pour Gaza et la Cisjordanie a présenté, mercredi 5 mars à Jérusalem, les conclusions du rapport d'étape soumis aux donateurs à Londres, en février. Rien ne semble pouvoir arrêter la destruction de l'économie palestinienne, qui compte désormais plus de $50 \%$ de chômeurs, alors que plus de $60 \%$ des Palestiniens ( 2 millions de personnes) vivent sous le seuil de pauvreté fixé à 2 dollars (environ autant d'euros) par jour et par personne. (Le Monde, vendredi, 7 mars $03 / 3$ )

L'examen des ajustements déictiques entre les deux segments du DR, nous a conduite à distinguer non un seul, mais trois cas de figures. Dans le premier, le cadre énonciatif de la Cit n'est pas adapté à celui du DC. La rupture syntaxique va donc de pair avec la rupture énonciative, comme en (5) où le passé composé du DC s'associe au présent et, surtout, au futur (souligné) du segment Cit:

(5) Tout en se refusant à répondre à «des questions hypothétiques » sur l'attitude de l'Allemagne au Conseil de sécurité en cas de vote sur d'éventuelles sanctions militaires contre Saddam Hussein; il a répété les trois principes qui fondent la position de son gouvernement : premièrement ; la résolution 1441 doit être strictement appliquée ; deuxièmement, tout doit être fait pour empêcher la guerre ; troisièmement, l’Allemagne ne participera pas à une intervention armée. (Le Monde, mercredi, 15 janvier 03/2)

Dans le second cas, les ajustements temporels et ceux d'autres déictiques (pronoms personnels, adverbes) entre la phrase introductive et la Cit reformulation sont neutralisés: les pronoms personnels qui interviennent dans les contextes retenus appartiennent à la troisième personne grammaticale, les adverbes sont absents et le temps qui domine est le présent. Par conséquent, l'insertion d'une Cit dans n'importe laquelle de ces séquences semble particulièrement facile :

(6) Ariel Sharon le répète: la guerre qui se prépare contre l'Irak ne concerne pas directement l'Israël. (Le Monde, vendredi, 21 février 03/1)

Le troisième cas de figure est représenté par des séquences dont la Cit s'adapte au cadre énonciatif du DC, par exemple :

(7) Le ministre avait annoncé ses intentions dès juillet 2002 : la France allait procéder à des retours forcés collectifs et se disait prête à les organiser avec d'autres pays européens dans le cadre de la lutte contre l'immigration clandestine. (Le Monde, vendredi 7 mars $03 / 6$ )

Le premier emploi, comportant une Cit non typographiée, mais intervenant dans la séquence sans transpositions de déictiques, comme en (5), nous a semblé au premier abord avoir trait au modèle du DD interprétatif, ou plus précisément au discours direct libre ( DDL) puisqu'il semble imiter certaines de ses caractéristiques: phrase introductive, deux-points, indépendance énonciative de la Cit. Dans la conception globalisante du DR, le DDL, pris au sens de «sans mention de verbe déclaratif ${ }^{15}$ ne s'adapte pas aux occurrences relevées dans notre corpus présentant comme DC une phrase introductive type. Mais si on comprend le discours direct libre au sens de " sans marqueurs typographiques de la reproduction » mais aussi au sens de 《 qui garde son cadre énonciatif ${ }^{16}$, apparemment, on est plus près du modèle. Apparemment, car à notre sens, on n'est certainement pas dans le modèle du DDL : pris ensemble, l'absence de guillemets-italiques et le signal de la résomption du dire (ou de son rapport approximatif) dans le DC ne collent pas avec l'image du direct. Par ces traits, la séquence analysée actualise deux signaux très forts de la reformulation : formel (absence de typographie) ${ }^{17}$ et sémantique (verbes de parole/ses équivalents). Sur le fond, on peut admettre que ces séquences du DR se rangent parmi les reformulations et que la non transposition des déictiques n'est qu'un jeu énonciatif. Les propos reformulés (parfois à valeur nettement illocutoire) sont saisis non pas par rapport au point de repère fourni par le DC, donc celui du rapporteur, mais par rapport au moment de la lecture. Le dire cité fait dès lors partie de l'actualité du lecteur. Du coup, les espaces temporels de l'EC et des lecteurs 
coïncident. Néanmoins, la séquence entière renferme, paradoxalement du point de vue de la vulgate, une reformulation non homogène. L'ordre des niveaux énonciatifs du DR est brouillé. La séquence évolue entre le modèle du direct (DD) lui empruntant de faibles marques de reproduction (non transposition de cadre énonciatif) et celui de l'indirect (DI) dont elle tire les traits sémantiques (signal de la résomption du dire) et formels (absence de la typographie) de la reformulation. D'après ces constatations, nous pensons qu'il est judicieux de parler dans ce cas de discours «mixtes » du DR, au sens d'une sorte de mosaïque de traits propres aux DD/DI. Serait-ce une sorte de discours indirect libre (désormais DIL) basculant vers le DD?

Dans le second contexte examiné (6), en quelque sorte « intermédiaire » par rapport aux deux précédents, les segments Cit, annulant pour ainsi dire les ajustements déictiques, rapprochent les séquences du DR dont elles font partie des types d'emplois que Marnette appelle discours neutralisés (par opposition au discours direct et indirect qui s'inscrivent dans la dichotomie «sans /avec ajustements ») (2004: 56). S'alignant sur la même logique de neutralisation, Komur propose de parler d'archiformes du DR (2004b : $71-72$ et 2004a : 56). Nos séquences neutralisées correspondraient-elles au cas du DIL dont les «indices se diluent dans le contexte » selon l'expression de Bally (infra)?

En examinant les DR illustrés par le dernier groupe de séquences (7), nous avons pensé les associer aux formes du DIL. Le DIL est d'habitude ${ }^{18}$ considéré comme un cas particulier de DI se caractérisant par l'ellipse du verbe de parole et de la conjonction de subordination « que ». Ce discours est donc libre car non subordonné à un verbe de parole, et il est indirect car il garde les transpositions grammaticales des personnes et des temps. Charles Bally, l'un des premiers à traiter du DIL en linguistique, l'a placé en grammaire à côté du duo consacré DD et DI. Bally reconnaît pourtant qu'il arrive que les indices grammaticaux se diluent dans le contexte narratif et que le style indirect «ne ressorte que de la situation » (1912 : 456-457). C'est de ce point de vue que le DIL est souvent présenté comme une intrusion de la parole dans le récit et comme une forme très ambiguë, parfois trop opaque pour pouvoir être classée parmi les objets de recherche de la linguistique. Allant à l'encontre des ces tendances, Vuillaume (2000) ou Mellet ( 2000), entre autres, insistent sur la signalisation ou les marqueurs du DIL, comme, à titre d'exemple, les temps grammaticaux en « -ait », le pronom « on » dans certains de ses emplois, des fragments dénotant une activité langagière (associables pour nous aux VP des DC), etc. D'autres chercheurs, comme par exemple Vetters soulignent la transparence de l'énorme majorité des emplois du DIL (1989: 67). Pour nous, on peut placer les occurrences analysées dans ce paragraphe parmi ce type d'emplois. Nous pensons donc que le DIL, comme toute autre forme du DR, peut être considéré comme une séquence textuelle bisegmentale, contenant le DC et la Cit ${ }^{19}$. Le DC de nos séquences de DIL (7) est relativement univoque : il contient un VP ou son équivalent, il identifie l'énonciateur cité et se termine par une marque typographique - par les deux-points qui signalent le passage à la Cit au même degré que la typographie de la phrase introductive typique du $\mathrm{DD}^{20}$. Une telle variante du DIL se caractériserait, d'une manière univoque, et par les transpositions déictiques, et par l'absence de subordination conjonctive.

\subsection{Phrase introductive/complète + Citation mixte}

Les séquences de ce contexte distributionnel ne diffèrent pas beaucoup, en apparence, de celles de la combinatoire précédente : leurs DC contiennent souvent un VP dont le sens se ramène à une sorte de résumé des dires ou signalent le rapport approximatif (« en substance ») des propos. Ensuite, c'est le segment Cit mixte qui intervient, soit après les deux points (8), soit après un point final qui ouvre sur une nouvelle phrase (9) :

(8) Ephraïm Halévy, ancien directeur du Mossad, a été le premier à théoriser tout haut ce qui se dit tout bas au sein du Planning militaire israélien: un renversement du régime irakien aura nécessairement un "effet de dominos » sur l'ensemble de la région. (Le Monde, vendredi, 21 février 03/2)

(9) M. Chadelat a présenté aux partenaires sociaux, fin décembre 2002, les grandes lignes d'un nouveau système de remboursement des soins à trois étages qui fait davantage appel au privé. L'Etat s'engagerait à « assurer le meilleur accès aux soins 


\begin{abstract}
à tous les Français » par le biais d'une " couverture maladie généralisée » assurée à la fois par la "Sécu» et une "assurance-maladie complémentaire de base ». Ce deuxième étage - facultatif - serait en partie financé par l'Etat à travers un "chèque » santé dégressif suivant les revenus des assurés (environ $8 \%$ des Français n'ont pas de complémentaire). ( Le Monde, vendredi, 21 février 03/7)
\end{abstract}

(10) Pour Washington, les choses paraissent entendues : la guerre est inévitable, sauf si Saddam Hussein « désarme ou s'en va». (Le Monde, vendredi, 7 mars 03 /1)

Pour interpréter les séquences (8-10), nous nous proposons de rester dans la logique des distinctions appliquées supra. Les occurrences relevées soit dissocient les cadres énonciatifs entre le DC et la Cit (8), soit les ajustent (9) ou les neutralisent (10).

Abstraction faite pour l'instant des îlots textuels, la séquence (8) s'apparente, à notre sens, aux discours libres mixtes, la séquence (9) au DIL et l'exemple (10), étant neutralisé, peut être associé à un DIL " dilué », donc aux archiformes. Les îlots textuels qui s'interposent dans les Cit reclassent ces séquences en variantes appelées par Bakhtine «verbalo-analytiques» (1977: 179) et par Authier, "quasitextuelles » (1978: 73) : variantes illustrant l'attitude spécifique du rapporteur qui, reformulant les propos des autres, en garde certaines expressions intactes. En bref, les séquences entières relèveraient du DIL « verbalo-analytique », ou du discours libre mixte « verbalo-analytique ».

Pour décrire certains emplois des îlots textuels, Maingueneau (1994 : 133) propose le terme et le concept de résumé avec citations : pour lui, c'est une forme de DR employée aussi bien dans la presse que dans les écrits académiques et qui consiste en une reformulation condensée qui restitue en principe le point de vue du locuteur cité. Les paroles citées sont mises à la fois entre guillemets et en italiques comme dans l'exemple suivant :

(11) $\mathrm{X}$ a tenu une conférence de presse hier. La France "ne se désintéresse pas de la situation" mais veut "prendre ses distances avec ses alliés". Elle est ouverte "aux nouvelles propositions" de ses partenaires. » (Charaudeau, Maingueneau $2002: 193$ ).

A nos yeux, l'exemple (11) est associable aux séquences du DR recueillies dans ce paragraphe. Il peut être analysé en tant qu'effet de montage du DC (souligné) qui contient un signal de prise de parole équivalent du VP, c'est-à-dire un nomina dicendi qui est un condensé nominal générique (« une conférence de presse »). Le segment Cit mixte arrive après le point et embrasse deux phrases. Puisque l'occurrence relève des cadres énonciatifs dissociés, elle ne serait pour nous qu'une version verbaloanalytique du discours libre mixte ( $c f$. Biardzka, 2009). La démarche de Maingueneau est différente : pour lui, le critère prioritaire dans l'analyse de ces séquences est l'intrusion des îlots textuels dans les Cit.

On voit bien d'après ces exemples que la nature même des séquences représentant la parole d'autrui dans la presse est qu'elles sont, toujours, ou presque toujours, l'effet d'une réduction, d'un choix. En fait, la diffusion de l'intégralité des discours, même si elle est souhaitable, est généralement impossible à cause du budget-temps-espace et du budget-attention réduits des journalistes et, aussi, du public.

\title{
2.4 Cit reproduction + incise
}

L'agencement de la Cit reproduction avec incise postposée est une variante du DD qui n'est pas oubliée des grammaires. Souvent mentionnée ${ }^{21}$ par la vulgate, elle apparaît régulièrement dans notre corpus :

(12) «Ségolène Royal a compris qu'elle ne pourrait rien faire sans le parti et eux ont compris qu'ils ne peuvent pas gagner sans elle », estime un proche. (Le Figaro, samedi11-dimanche 12 septembre $10 / 3$ )

(13) "Pourquoi les femmes sont-elles exclues de certains métiers, comme l'artisanat? », dit-elle. (Le Monde, mercredi, 5 mars 03/11) 


\subsection{Cit reformulation + incise}

Les emplois de l'incise, qui s'intègre facilement dans nombre de contextes «libres» du DR, ont beaucoup attiré l'attention des chercheurs ( $c f$. Rosier, $1999: 247$ et plus particulièrement 257-266 ; Lips, 1926 : 48-56 ; Banfield, 1973 ; Komur, 2004b : 71-72). Nous venons d'aborder supra ses occurrences «classiques » au DD. Selon l'opinion de beaucoup de chercheurs, surtout Bally (1912: 598) et Lips (1926 : 48-56), les emplois de l'incise avec transpositions déictiques relèvent du $\mathrm{D}(\mathrm{S}) \mathrm{IL}^{22}$. Comme le signale Rosier (1999 : 258), d'autres encore, comme Banfield (1973), Cornulier (1978) et Rey-Debove (1983) reclassent ce type d'emplois en D(S)IL.

Paradoxalement, notre corpus ne contient pas ce type d'occurrences, amplement décrites par la théorie grammaticale. Ceci confirmerait la thèse de Rosier sur la théorisation par excellence « littéraire » (à partir des emplois littéraires) du DIL, qui associe « un usage particulier de l'imparfait conjoint au passé simple dans la construction du récit » $(1999: 281)$. En fait, les séquences que nous avons recueillies dans le corpus semblent s'éloigner du modèle consacré de Bally, cependant, sans le contredire du tout. Elles s'inscrivent dans certaines des régularités d'emplois que nous avons déjà analysés. Amplement représentées dans le corpus, les séquences de DR avec incise finale ne contiennent pas d'ajustements déictiques: la Cit garde son propre cadre énonciatif et ce sont surtout les temps grammaticaux qui le signalent.

Ce type de séquences tend vers les discours libres mixtes: les marques du rapport « en substance », sont nettes, même si elles sont souvent contextuelles. Nous pensons surtout à l'exemple (14) où le pluriel attribué au substantif désignant l'énonciateur cité prévaut sur le caractère résomptif de la Cit: non typographiée, elle représente un certain résumé du scoop et subsume les émissions des télévisions du monde entier. En fait, tous les emplois que nous avons repérés proviennent des sources du desk ${ }^{23}:$ ce sont surtout des filets. Le journaliste du desk essaie toujours de « façonner », récrire une nouvelle brute, il peut procéder aussi à une « mouture » d'information : la construire à partir de plusieurs sources. En voici des exemples :

(14) Caracas. Le président de Fedecámaras, la fédération vénézuélienne, Carlos Fernández, l'un des principaux opposants au président Hugo Chávez, a été arrêté dans la nuit du mercredi 19 au jeudi 20 février par la police politique (Disip), ont annoncé les télévisions. (Le Monde, vendredi 21 février 03/4)

(15) Omc: Washington a renoncé à porter devant l'Organisation mondiale du commerce son différend avec l'Union européenne sur les organismes génétiquement modifiés (OGM), dont les 15 refusent l'importation, a annoncé jeudi 20 février, un porte-parole de l'ambassade américaine à Londres. (Le Monde, vendredi 21 février $03 / 4)$

(16) Alors que les pays donateurs d'aide alimentaire débattent de l'effet dissuasif de l'étranglement de la Corée du Nord, ce sont 6 à 8 millions de personnes dont la vie est en danger, a déclaré la semaine dernière à Pékin Maurice Strong, envoyé du secrétaire général de l'ONU, de retour de Pyongyang. ( Le Monde, jeudi, 23 janvier 03 /4).

\subsection{Cit mixte + incise}

A côté de la Cit reproduction et de la Cit reformulation, la Cit mixte se combine aussi régulièrement avec l'incise finale. Remarquons l'occurrence de «dixit», en (17), qui, comme l'observe Rosier, « oscille entre une pesée critique de l'ordre de prétendre et un usage argumentatif, affirmatif» (1999: $213)^{24}$. Le « dixit » s'associe toujours à la Cit reproduction, qu'elle soit intégrale ou partielle :

(17) De leur côté, le PS, le PCF et la LCR refusent de «servir de marchepied à M. Bayrou », dixit Bruno Le Roux, secrétaire national du PS chargé des élections. (Le Monde, jeudi, 6 février 03/9)

(18) L'accord avec l'Union nationale « signifie que le prochain gouvernement [israélien] sera incapable d'adopter une politique équilibrée sur le processus de paix. Il ne laissera plus aucune chance à la paix ", a assuré l'un des principaux 
négociateurs palestiniens, Saëb Erakat, qui a même prédit une réoccupation prochaine de la bande de Gaza. ( Le Monde, jeudi, 27 février 03/3)25

(19) Il n'est pas question de jouer "à cache-cache », a déclaré M. Bush devant des responsables du Parti républicain réunis à White Sulphur Springs, une station thermale de Virginie occidentale. (Le Monde, mardi, 11 février 03/)

(20) Sur le fond, le bilan est lourd et la machine pénale "encore malade", constate le procureur général Favara : huit crimes ou délits sur dix restent impunis. (Le Monde, mercredi, 15 janvier 03/5)

(21) «Nous pensons que, à la fin du mois, il sera largement prouvé que l'Irak ne coopère pas " comme le requiert la résolution 1441 des Nations Unis, a affirmé le secrétaire d'Etat américain, Colin Powell, dans un entretien accordé à cinq journalistes de pays qui viennent d'entrer au Conseil de sécurité des Nations unies en tant que membres non permanents. (Le Monde, dimanche, 19/ lundi, 20 03/2)

Lors de l'analyse de ces séquences de DR, nous avons été particulièrement attirée par les propriétés énonciatives des Cit mixtes relevant de leur combinatoire avec le segment DC-incise. La mixité est d'habitude décrite comme l'intrusion d'îlots textuels dans un segment reformulé ( $c f$. à ce propos Authier, 1978 : 73-74, Rosier, 1999 : 233-237). Par conséquent, les expressions guillemetées soit s'adaptent au cadre énonciatif du rapporteur (emploi considéré comme régulier en modalisation autonymique), soit gardent leur propre cadre (cas de l'hybridation «citationnelle»). A nos yeux, un sens inverse de l'insertion est possible. Dans le contexte du DR avec incise, on aurait parfois affaire à l'intrusion de segments reformulés dans les reproductions. Ainsi, les Cit reproductions mixtes consisteraient à intégrer des Cit reformulations dans la Cit reproduction. Leurs déictiques sont ajustés au cadre énonciatif de l'énonciateur cité et non pas à celui du rapporteur et on ne peut pas parler, à cette occasion, d'une hybridation quelconque. Dans ce cas, le rapporteur journaliste rend compte du discours autre en reproduisant la matérialité des propos d'origine tout en y mêlant des reformulés, comme dans les exemples suivants ${ }^{26}$ :

(22) Pour le moment, "il est clair, pour moi, qu'il ne désarme pas », a répété M. Bush. ( Le Monde, jeudi, 23 janvier 03/4)

(23) « Nous voulons croire » qu'il est "possible » d'adopter une position commune contre une "action unilatérale " à l'encontre de l'Irak lors de la réunion des ministres des affaires étrangères de l'UE prévue lundi et mardi, a déclaré M. de Villepin lors d'un point de presse (...) ( Le Monde, jeudi, 23 janvier 03/4)

A ce qu'il nous semble, ces séquences entières peuvent s'interpréter en termes de DD pseudo-textuel (ou DDL): le segment Cit a un caractère manifestement pseudo-textuel : il réunit les reproductions en les parsemant de reformulés.

Le dit « authentique », marqué par les guillemets et les italiques fonctionne souvent comme «preuve » destinée à cautionner différentes reformulations et augmenter la fiabilité du titre. Cependant, au moyen de ces mêmes signes typographiques, les journaux se distancient par rapport au dit rapporté et renforcent souvent cette distance par les incises. Ils construisent sa fiabilité et son prestige en plaçant les nouvelles d'actualité au centre de son discours pour assurer la rapidité et la «fraîcheur» de la diffusion de l'information.

\section{Combinatoire contrainte}

La séquence du DR « contraint» est sémiotiquement et syntaxiquement homogène. Cependant, du point de vue énonciatif, elle est hybride : c'est seulement le segment DC qui contient les propos propres au rapporteur-journaliste. Dans le segment Cit, ce même journaliste rapporte un contenu qui ne vient pas de lui. Distinguons d'abord les types principaux de constellations du DC et des Cit : 


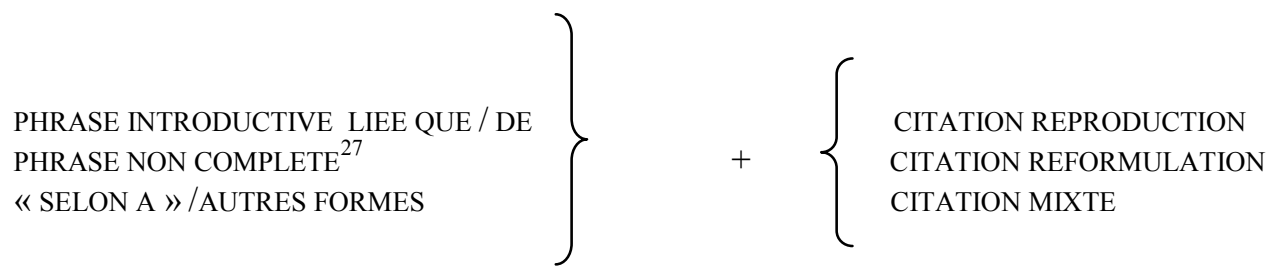

La phrase introductive peut être liée grammaticalement à la Cit à l'aide de deux régissants: à l'aide de la conjonction «que » ou de prépositions comme «de» et «à ». Les DC liés ouvrants ainsi représentés grammaticalement se combinent dans les articles journalistiques aussi bien avec des Citations reproductions qu'avec des Citations reformulations et Citations mixtes.

Nous avons choisi de montrer comment la phrase avec "que » se combine régulièrement à la reformulation, à la reproduction et à la Cit mixte selon le schéma suivant :

PHRASE INTRODUCTIVE LIEE /QUE $+\quad\left\{\begin{array}{l}\text { CITATION REFORMULATION } \\ \text { CITATION REPRODUCTION } \\ \text { CITATION MIXTE }\end{array}\right.$

\subsection{Phrase que + Citation reformulation}

Avec les séquences «phrase que + Cit reformulation », nous abordons une forme de DR reconnue et consacrée, largement décrite par les grammaires: le discours indirect (DI), considéré comme l'un des principaux schémas français de la transmission de la parole d'autrui. Pour Authier-Revuz, le «que », signe quasiment prototypique du DI, signale une reformulation-traduction des propos cités (1992: 6364) :

(24) Elle explique que sa ville doit se développer et a besoin d'argent pour préserver ses monuments. (Le Figaro, samedi 11 et dimanche 12 septembre 2010/2)

La majorité des séquences du DR inventoriées dans ce point illustrent parfaitement ce schéma. Cependant, nous avons repéré quelques cas (exemple 25) où les déictiques (temps grammaticaux) de la Cit n'avaient pas été ajustés au cadre énonciatif du DC. Dans ces cas, les propos reformulés seraient saisis non pas par rapport au cadre énonciatif du rapporteur mais par rapport au moment de la lecture. En outre, dans beaucoup de séquences, les ajustements sont en fait neutralisés (le présent, la troisième personne grammaticale). L'indirect «prototypique » montrerait-t-il en discours une facette de " mixité » en plus, ou nous retrouvons-nous ici plutôt devant une simple «déviance » grammaticale ? En voilà un exemple :

(25) M. Powell a indiqué, aussi, que depuis son exposé du 5 février au Conseil de sécurité, d'autres informations recueillies par les services de renseignement ont appris au gouvernement que les Irakiens ont déplacé des agents chimiques et biologiques, fin janvier, de Bagdad vers les régions frontalières de la Syrie et de la Turquie. (Le Monde, vendredi, 7 mars 03/2)

En fait, profondément centrés sur l'actualité, les journaux utilisent abondamment les temps " déictiques ", le passé composé et surtout le présent. Même s'ils reformulent, ils brouillent les cadres énonciatifs, « oublient » les transpositions déictiques et mettent en marche l'illusion du dit rapporté (donc passé), pourtant contemporain au moment de la lecture, palpable et proche du lecteur. 
Par rapport au DD, le DI, pris souvent au sens générique de "reformulation », "prête une oreille différente au discours d'autrui », comme le souligne Bakhtine (1977: 178). Selon le chercheur russe, le DI n'est pas un dérivé du DD, il est une façon à part de la transmettre les paroles d'autrui. Si le DD, par définition, permet de considérer l'énonciation d'autrui comme un tout compact: «inanalysable, immuable, (...) impénétrable et inerte » (Bakhtine, $1977:$ 176), le discours indirect se caractérise par une transmission analytique du discours d'autrui : l'analyse est « l'âme du discours indirect ».

\subsection{Phrase que + Citation reproduction}

Dans une certaine mesure, les séquences qui combinent la phrase avec conjonction «que » à la Cit reproduction semblent paradoxales. Le «que » est en fait une marque de reformulation par excellence alors que la Cit reproduction s'associe à l'idée de la littéralité, même si elle est souvent approximative. A nos yeux, le paradoxe est apparent. Certes, la conjonction «que» est toujours un signe de « reformulation-traduction » ou, comme le dirait Bakhtine, un signal d'analyse. Nous pensons que les séquences examinées, régies par «que», représentent simplement un degré minimum de « reformulation-traduction». Le « que » explicite ici un choix des propos cités, l'analyse ne consiste pas à « reformuler» au sens littéral, mais à «trier» des propos à citer. En dernière analyse, déclarer qu'on effectue un choix parmi différents propos à citer (à l'aide de «que ») ne revient-il pas à avouer une certaine reformulation de l'énonciation rapportée? L'effet discursif de ces démarches se marque d'ailleurs nettement par la typographie: les Cit reproductions qui suivent le « que » commencent par une minuscule, et le point final se place hors des guillemets. En bref, ces séquences contiennent une Cit reproduction présentée comme partielle, comme un fragment d'un énoncé plus ample. Les déictiques de ces séquences s'ajustent, dans la plupart des cas, au cadre imposé par le DC. Dans beaucoup de contextes, les propos évoqués sont neutralisés. Les séquences relèvent de la modalisation autonymique et sont un cas extrême de DI « quasi-textuel» (cf. Authier, 1978: 73-74), car la reformulation des propos d'origine est minime :

(26) A l'inverse, Me Merchat, pour les deux policiers suspects, répète qu' « aucun fait n'établit de faute disciplinaire ou pénale commise par quelque fonctionnaire que ce soit ». (Libération, samedi 11 et dimanche 12 septembre 2010)

Dans certains cas, on peut parler de véritables Cit «hybrides $»^{28}$, postposées aux phrases avec « que », juste comme dans la séquence (27), contenant une Cit reproduction dont les déictiques restent intacts et qui représentent simplement un degré de reformulation ultra-minimal :

(27) Interrogé par CNN, l'ancien président Bill Clinton a estimé que «sur le plan militaire, nous pouvons battre Saddam Hussein facilement et rapidement ». (Le Monde, vendredi, 14 février $03 / 2$ )

La raison d'être de l'« hybridation » a été amplement discutée par Komur (2003 : 131-145 et 2004b : 70). Rappelons que dans le cas des Citations hybrides ${ }^{29}$ les déictiques se calculent par rapport à deux cadres énonciatifs bien distincts : le premier est celui du journaliste, le second celui de l'énonciateur cité. A juste titre, Komur remarque surtout que l'ajustement déictique est souvent bloqué par le pronom «nous » (et sa série : «nos, notre $»)^{30}$. En fait, dans un discours reformulant, l'équivalent possible de ce déictique n'a pas toujours le même sens que la forme dans le discours d'origine. Le rapporteur journaliste le laisse donc intact. Ainsi, en (27) les transpositions automatiques relevant de la reformulation-traduction fabriquent soit une inadéquation de sens, soit directement un contresens par rapport à l'original. La référence de « ils » dans la partie reformulée est ambiguë : elle peut être prise soit au sens inclusif, soit au sens exclusif $^{31}$ alors que l'emploi de «nous » lève toute ambiguïté .

Dans la pratique du discours journalistique, le «que » introduisant la Cit reproduction peut être extérieur à cette Cit même et appartenir sans équivoque au DC. C'est le cas dans la majorité des emplois enregistrés dans le corpus ( $c f$. supra). Dans certains autres cas, le « que » peut être intégré à la Cit et, par là, exclu du DC comme dans l'occurrence (28) : 
(28) En réponse, le professeur Henrion a estimé, vendredi, "qu'il serait logique qu'un violeur conscient de sa séropositivité soit sanctionné par des peines plus lourdes ». (Le Monde, dimanche, 19/ lundi 20, janvier 03/8)

Il est vrai que, dans ce cas, les séquences de DR deviennent plus proches du DD. Ainsi, Rosier (1999: 217-221) considère que ces types de DR, par leurs marques différentes, s'approchent tantôt du DD (non ajustements déictiques possibles, traits de l'«oralité » gardés à l'écrit, le «que » extérieur au DC et absorbé par la Cit, etc.), tantôt du DI (ajustements déictiques effectués, traits de l'oralité effacés, le « que » extérieur à la Cit, etc.) au point qu'on puisse parler de DD avec «que » ${ }^{32}$ ou de DI «avec marques typographiques » (Rosier, 1999: 245). Puisque les différences entre les deux ne sont pas toujours faciles à discerner (ni même parfois discernables), elle opte pour la dénomination de « mixtes ».

\subsection{Phrase que + Citation mixte}

La phrase avec «que » est, dans le DR journalistique, régulièrement suivie de Cit mixtes. Ces segments Cit, en partie présentés comme reformulés, contiennent des îlots textuels, intégrés dans la syntaxe du segment. Les guillemets encadrent souvent les mots isolés comme en (31) ou des syntagmes très peu développés, comme en (30). Dans ces cas, on a l'impression que la typographie intervient aléatoirement et n'a pas de contenu réellement pertinent, mais vise plutôt à atteindre un effet crédible et authentique de « nouvelles d'actualités »:

(29) Dans son fameux discours du Caire, en juin 2009, Barack Obama avait déjà prévenu que le «cycle de méfiance et de discorde» entre l'Amérique et l'Islam ne serait pas simple à " briser ». (Libération, samedi 11 et 12 dimanche 2010/2)

(30) Mme Touraine nous a précisé qu'elle n'avait "pas revendu [ses] actions ».

( Le Monde, samedi, 25 janvier 03/19)

(31) Le général Myers a assuré que des efforts « extraordinaires» seront faits pour épargner les civils, mais il n'a pas caché que des « gens vont mourir». (Le Monde, vendredi , 7 mars $03 / 2$ )

Comme nous l'avons établi, les Cit mixtes consistent ici à cumuler la Cit reformulation et la Cit reproduction. Dans le contexte distributionnel présenté, le rapporteur journaliste rend compte du discours autre en reformulant le propos d'origine tout en y mêlant des mots, des expressions ou même des propositions entières qu'il présente comme réellement prononcées par l'énonciateur cité. Rappelons que cette tendance du rapport des paroles d'autrui, décrite encore par Bakhtine (1977:179) et qualifiée de variante « verbalo-analytique » du DI réunit, selon le chercheur russe, une décomposition analytique du sens objectif et une indication de l'expression qui laisse intacts certains mots et certaines tournures du discours d'autrui. Pour sa part, comme nous l'avons mentionné supra, Authier parle de DI «quasi textuel» $(1978: 73)$.

Certaines transpositions déictiques à l'intérieur des segments Cit attirent une attention particulière. Ainsi, en (30), les « sous-marins » énonciatifs ([ses]), pour reprendre le terme pittoresque de Rosier (2002 : 31), eux-mêmes intégrés dans la syntaxe de la Cit reproduction partielle, ont pour fonction d'adapter le cadre énonciatif de l'EC à celui du rapporteur. Comme nous l'avons déjà vu, en discours, la reproduction et la reformulation ont toutes deux un caractère graduel. Certaines Cit reproductions sont présentées comme intactes, certaines autres subissent des modifications flagrantes. L'exemple (31) présente un degré de « quasi-textualité » particulièrement développé et intéressant. Le « que »s'y associe au segment Cit mixte qui garde le cadre temporel de l'EC: aussi bien par rapport à sa partie «reformulée» (« seront faits ») que par rapport à sa partie « reproduite » (« vont mourir »).

En fait, la tâche du rapporteur ne consiste pas à s'adapter aux modèles canoniques du rapport de la parole d'autrui. Le journaliste s'adapte plutôt à la réalité crue et aux besoins du contrat de communication. De la sorte, il peut « recréer une parole à la place de mots qu'il n'a pu retenir «par coeur» ( $c f$. Authier, 1978: 76), ou arranger « un peu» des propos qu'il croit répétitifs ou lassants ». Conformément aux prescriptions rédactionnelles du journal, dès qu'il trouve que ce qu'il a construit 
comme segment textuel Cit est très proche du discours d'origine, il peut le marquer par les guillemets. Dès qu'il estime que sa Cit s'éloigne de l'original, il les élimine. La reproduction et la reformulation ont donc, en discours, un caractère graduel et évaluatif. Ce n'est pas au grammairien de décider des propriétés (littéral/reformulé) des segments textuels. C'est le rapporteur qui en décide.

\section{CONCLUSION}

Le discours grammatical dresse depuis toujours un inventaire autant que possible exhaustif de structures générales, de formes et de paradigmes abstraits dont, depuis de Saussure, nous avons pris l'habitude de dire qu'ils font partie du système de telle ou telle langue naturelle et qu'il est possible ensuite de les retrouver ou de les utiliser comme incarnations concrètes dans l'organisation de tel texte ou type de texte particuliers. Ce ne sont que des (proto)-types, des paradigmes exhibant ce qu'il y a de général, de transindividuel dans une forme. Compris de la sorte, le terme de discours rapporté ne renvoie pas au champ empirique large des séquences textuelles rapportant certains "événements énonciatifs ». Ainsi, notre démarche de voir le DR comme effet du montage de deux segments DC + Cit, montre une diversité d'emplois assez régulière qui se matérialise en discours. Ainsi, à quelques exceptions près, les DC, que ce soit en combinatoire libre ou contrainte, se présentent systématiquement avec tous les trois types de Cit de base (reproductions, reformulations et mixtes). C'est de là que vient le nombre croissant de DR. La tendance est augmentée par les possibilités grammaticales évidentes des modifications de l'ordre des segments DC et Cit (DC+ Cit mais aussi Cit + DC), ainsi que par leur multiplication dans le discours (par exemple du type DC $+\mathrm{Cit}+\mathrm{DC}$ qui augmente le nombre de segments $\mathrm{DC}$, ou du type Cit $+\mathrm{DC}+\mathrm{Cit}$ qui répète les segments $\mathrm{Cit}$ ). En fait, dans chacune des occurrences, différentes marques (sémanticoénonciatives, pragmatiques et, aussi, formelles) s'actualisent et émergent ou bien s'effacent et disparaissent. Dans un inventaire comme celui que nous avons entrepris de dresser, nous avons essayé de tenir compte d'une part d'une quantité importante de variantes du rapport du dit dans un discours analysé, et d'autre part, de leur éventuelle gradualité dynamique, d'un certain continuum. Ainsi conçu, l'inventaire permet de spécifier les tendances du discours journalistique dans la dynamique de la représentation du discours autre.

\section{Références bibliographiques}

Authier, J . (1978). Les formes du discours rapporté. Remarques syntaxiques et sémantiques à partir des traitements proposés. DRLAV 17, 1-87.

Authier-Revuz, J. (1992). Repères dans le champ du discours rapporté. L'information grammaticale,55, 38-42.

Authier-Revuz, J. (1993). Repères dans le champ du discours rapporté (suite). L'information grammaticale, 56, 1015.

Authier, J. (2004). La Représentation du discours autre : un champ multiplement hétérogène. In Lopez Muñoz J.M., Marnette S. et L. Rosier L. (éds), Le discours rapporté dans tous ses états. Paris : L'Harmattan, 35-53.

Bakhtine, M. (1977). Le marxisme et la philosophie du langage, traduit du russe par Marina Yaguello, Paris : Minuit. Première édition russe publiée sous la signature V.N. Volochinov (1929), Marksizm i filozofiya yazyka, Leningrad.

Banfield, A. (1973). Le style narratif et la grammaire des discours directs et indirects. Change, 16-17, 190-226.

Bally, Ch. (1912). Le style indirect libre en français moderne. Germanisch-Romanische Monatsschrift 4, 549-556, 597-606.

Bally, Ch. (1914). Figures de pensée et formes linguistiques. Germanisch-Romanische Monatsschrift 6, 405-422, 456-470.

Bally, Ch. (1944). Linguistique générale et linguistique française. Paris : PUF. 
Biardzka, E. (2009). Les échos du «Monde ». Pratiques du discours rapporté dans un journal de la presse écrite. Wrocław : Wydawnictwo Uniwersytetu Wrocławskiego.

Biardzka E . (2010). Différentes facettes de la mixité des discours rapportés, In Neveu F., Muni Toke V., Durand J., Klingler T., Mondada L., Prévost S., Paris, $2^{\mathrm{e}}$ Congrès Mondial de Linguistique Française, La Nouvelle-Orléans, 12-15 juillet 2010, Institut de Linguistique Française, accès en ligne : http://www.linguistiquefrancaise.org/index.php, consulté le 20.12.2010.

Bruña Cuevas, M. (1996). Le discours direct introduit par Que. Le Français Moderne LXIV, 1, 28-50.

Charaudeau, P., Maingueneau, D. (2002). Dictionnaire d'analyse du discours. Paris : Seuil.

Cornulier, B. de (1978). L'incise, les parenthétiques et le signe mimique. Cahiers de linguistique 8, Presses de l’Université de Québec, Montréal, 53-95.

Cunha, D. A.C. (1992), Discours rapporté et circulation de la parole. Louvain-la Neuve : Peeters.

Greilsamer, L. (réd.) (2004), Le style du « Monde ». édité par la Société éditrice du Monde (SAS).

Hausmann, F. J. (2000), La langue de la presse, In Antoine G., Cerquiligni B. (réd.) Histoire de langue française 1945-2000, Paris : CNRS 199-210.

Komur, G. (2003), Quelques réflexions autour des formes hybrides dans la presse française contemporaine. Roczniki Humanistyczne, tom LI, 131-145.

Komur, G. (2004a). L'îlot textuel et la prise de distance par le locuteur dans le genre journalistique . In LopezMuñoz J.-M, Marnette S. et Rosier L.(éds), Le discours rapporté dans tous ses états. Paris : L'Harmattan, 5463.

Komur, G. (2004b). Les modes du discours rapporté dans la presse et leurs enjeux polyphoniques . Pratiques 123$124,57-74$.

Lips, M. (1926). Le style indirect libre. Paris : Payot.

Maingueneau, D. (1994). L'énonciation en linguistique française. Paris : Hachette.

Marnette, S. (2004). L'effacement énonciatif dans la presse contemporaine . Langages 156, 51-64.

Mellet, S. (2000). A propos de deux marqueurs de bivocalité . In Mellet S. et Vuillaume M. , Le Style indirect libre et ses contextes. Amsterdam - Atlanta, GA : Rodopi, 91-106.

Meteva, E. (2002). La Citation journalistique avec ou sans guillemets, Faits de Langues 19 , 117-125.

Perret, M. (1994). L'énonciation en grammaire du texte. Paris : Hatier.

Rey-Debove, J. (1983). Le métalangage dans le langage parlé , Recherches sur le français parlé. Cahiers du Gars 5 , 211-226.

Riegel, M., Pellat, J.-C., Rioul, R. (1994). Grammaire méthodique du français. Paris : PUF.

Rosier, L. (1999). Le discours rapporté. Histoire, théorie, pratiques. Paris, Bruxelles : Duculot.

Rosier L. (2002), La presse et les modalités du discours rapporté : l'effet d'hyperréalisme du discours direct surmarqué , L'information grammaticale 94, 27-32.

Simonin, J. (1984). Les plans d'énonciation dans Berlin Alexanderplatz de Doblïn ou de la polyphonie textuelle. Langages $73,30-56$.

Strauch, G. (1974). De quelques interprétations récentes du style indirect libre. RANAM. Recherches anglaises et américaines VII, revue annuelle, 40-73.

Strauch, G. (1984). De la "littéralité" du discours rapporté. RANAM. Recherches anglaises et américaines XVII, revue annuelle, 159-181.

Vetters, C. (1989). Le Style Indirect Libre. In Vetters C., Temps et Discours, Anvers : Universiteit Antwerpen (=Antwerp Papers in Linguistics 59), 30-70.

Vuillaume, M. (2000). La signalisation du style indirect libre, In Mellet S. et Vuillaume M. Le style indirect libre et ses contextes, Amsterdam - Atlanta, GA : Rodopi, 107-130. 
Wilmet, M. (1998). Grammaire critique du français, Ilème édition, revue et corrigée. Paris-Bruxelles: Hachette/Duculot.

${ }^{1} \mathrm{La}$ présente étude est réalisée dans le cadre du projet de recherche $\mathrm{n}^{\circ} 4498 / \mathrm{PB} / \mathrm{IFR} / 11$ subventionné par le Narodowe Centrum Nauki (Centre National de Recherche) en Pologne. Niniejszy tekst został opracowany w ramach prac naukowych finansowanych ze środków budżetowych na naukę w latach 2011-2014 jako projekt badawczy $\mathrm{Nr}$ 4498 /PB/IFR/11.

${ }^{2}$ La prolifération des termes et des concepts en DR est surtout critiquée par Authier-Revuz (2004 : 35) qui plaide pour la simplification classificatoire des formes du DR. De la sorte, elle propose de traiter le discours narrativisé non pas comme un modèle du DR à part, mais tout au plus comme une variante discursive du discours indirect. Pour Strauch (1984: 173) et pour Perret (1994: 101) cette forme n'appartient pas au champ du DR. De même Cunha de Arruda (1992 : 66) souligne la fluidité des critères de la délimitation du discours narrativisé. Pour Wilmet (1998: 446), par exemple le discours direct libre est une forme qui s'installe assez mal parmi les modèles du DR et peut être associée au discours indirect libre dans une catégorie commune. Pour Authier, elle n'est qu'une variante non marquée du discours direct (2004:36).

${ }^{3}$ Haussmann souligne pour sa part que les journalistes «se battent - et ce alors qu'ils sont constamment dans l'urgence - sur le front d'un écrit que la société non professionnelle a de plus en plus de mal à maîtriser ». Mépriser leur travail est, selon lui, un jeu trop facile. Il avoue qu'il considère les journalistes comme «d'extraordinaires artisans - parfois artistes - de l'écrit et comme de précieux documentalistes de l'oral » (2000: 207-208).

${ }^{4}$ C'est peut-être le bon moment pour rappeler le principe de l'attitude descriptive en linguistique: c'est l'usage qui est le maître et le souverain de toute langue.

${ }^{5}$ La localisation dans le journal sera donnée entre parenthèses à la fin de chaque exemple. Elle contiendra la date de parution du numéro dépouillé ainsi que la page, suivant le modèle: ( Le Figaro, mercredi, 5 mars 03/8).

${ }^{6} C f$. à ce propos Maingueneau (1994) et Rosier (1999) qui emploient également le terme discours citant.

${ }^{7}$ L'analyse que nous présentons dans cette étude développe et remanie les idées contenues dans Biardzka (2009).

${ }^{8}$ Nous avons essayé de décrire ces critères dans Biardzka (2009).

${ }^{9}$ Evidemment, le terme n'est pas nouveau : il est utilisé dans les grammaires comme, pour ne citer qu'elle, celle de Riegel, Pellat et Rioul (1994: 598). Cependant, une remarque reste à faire : nous ne prenons pas ce terme au sens générique comme le fait ce manuel en indiquant : «Cette phrase peut occuper trois positions : avant (...), à l'intérieur ou après le fragment du discours direct (...) (1994 : 598). Pour nous, ce terme se rattache à une forme grammaticale précise et concrète.

${ }^{10}$ Abstraction faite pour l'instant de la rupture sémiotique et énonciative.

${ }^{11}$ Toutefois, il convient de remarquer que la syntaxe « interne » des DC transphrastiques dépend de leur position par rapport à la Cit : dans les cas de postposition et d'insertion, l'inversion du sujet est obligatoire.

${ }^{12}$ Sauf dans le cas du locuteur autodiégétique, difficilement trouvable dans le discours journalistique, qui cite ses propres dires.

${ }^{13}$ Appelée souvent proposition incidente ou incise terminale.

${ }^{14}$ A une exception près, citée par Rosier : si on classe les structures du type «comme (le) dit X » comme incises, celles-ci peuvent bien apparaitre en position initiale du DR (cf. 1999 : 250-251).

${ }^{15}$ Comme chez Bally (1944 : 50-51), ou chez Strauch (1974 : 424, 1984 : 176-177). Voir aussi à ce propos Rosier 1999 : 266-270).

${ }^{16}$ Comme chez Simonin (1984: 32) ou Meteva (2002:118). 
${ }^{17}$ Dans une séquence prise comme reformulée, les deux points sont interprétables aussi comme signe d'explication ( « c'est-à-dire ») et non de citation (« je cite »).

${ }^{18}$ C'est ainsi que Bally définit le discours indirect libre (qu'il appelle style indirect moderne) (1912: 553). Cette définition est retenue, entre autres, par Authier (1978: 77).

${ }^{19}$ Si cette forme du rapport de la parole d'autrui est souvent considérée comme vague, c'est parce que la délimitation des segments qui la composent est parfois particulièrement difficile faute de marqueurs spécifiques.

${ }^{20}$ Le rôle des deux points est très important dans les emplois « interprétatifs » du DR.

${ }^{21}$ Référons-nous encore une fois au manuel de Riegel, Pellat et Rioul (1994 : 598) qui énumère trois positions de la «phrase introductive ».

${ }^{22}$ Lips utilise le terme original de Bally.

${ }^{23}$ Rappelons que le desk est un atelier de traitement de l'information où arrivent les informations provenant des agences de presse ou d'autres sources (par exemple des institutions publiques, des associations qui publient leurs communiqués)

${ }^{24}$ La forme « dixit » s'apparente au morphème « sic ». Ce dernier, absent de notre corpus, accentue la mise à distance par rapport au propos cité, insiste sur la dimension critique et, en tant que tel, est un «marqueur critique de littéralité » (Rosier, 1999 : 214).

${ }^{26}$ Nous avons traîté ce sujet dans un article à part (Biardzka, 2010).

${ }^{27}$ En combinatoire contrainte, la phrase non complète introduit par exemple de différentes Cit qui sont condensés de parole.

${ }^{28}$ A propos de tels emplois, le guide du Monde écrit ceci: « Proscrire des formulations telles que : Le premier ministre affirme que " nous avons pris la décision... " . (Greilsamer, 2004 : 17).

${ }^{29}$ Komur n'emploie pas le terme « citation hybride », elle étudie les « formes » du DR en entier.

${ }^{30} \mathrm{La}$ quasi-totalité des occurrences que nous avons enregistrées dans notre corpus contiennent justement le pronom en question .

${ }^{31}$ Elle est alors en contresens flagrant par rapport au discours d'origine.

32 Le DD avec « que » a fait l'objet du travail de Bruña-Cuevas (1996) qui préconise de reconnaitre ces emplois comme forme légitime du DR, à côté des autres formes consacrées du rapport des paroles d'autrui. 